\title{
Range-separated hybrid and double- hybrid density functionals: A quest for the determination of the range-separation parameter
}

Cite as: J. Chem. Phys. 152, 244124 (2020); https://doi.org/10.1063/5.0010976

Submitted: 17 April 2020 . Accepted: 11 June 2020 . Published Online: 26 June 2020

Éric Brémond (D), Ángel José Pérez-Jiménez (D), Juan Carlos Sancho-García (D), and Carlo Adamo (D)

\section{Lock-in Amplifiers up to $600 \mathrm{MHz}$}

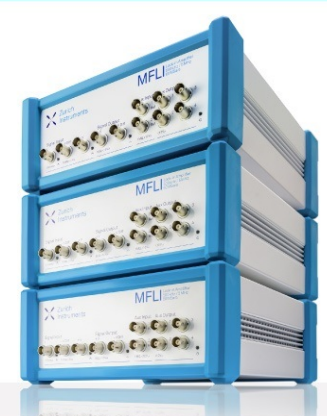




\title{
Range-separated hybrid and double-hybrid density functionals: A quest for the determination of the range-separation parameter
}

\author{
Cite as: J. Chem. Phys. 152, 244124 (2020); doi: 10.1063/5.0010976 \\ Submitted: 17 April 2020 - Accepted: 11 June 2020 • \\ Published Online: 26 June 2020
}

\begin{abstract}
Éric Brémond, ${ }^{1, a)}$ (D) Ángel José Pérez-Jiménez, ${ }^{2}$ (D) Juan Carlos Sancho-García, ${ }^{2}$ (D) and Carlo Adamo ${ }^{3,4}$ (D)
AFFILIATIONS

${ }^{1}$ Université de Paris, ITODYS, CNRS, F-75006 Paris, France

${ }^{2}$ Departamento de Química Física, Universidad de Alicante, E-03080 Alicante, Spain

${ }^{3}$ Chimie ParisTech, PSL Research University, CNRS, Institute of Chemistry for Life and Health Sciences (i-CLeHS), FRE 2027,

F-75005 Paris, France

${ }^{4}$ Institut Universitaire de France, 103 Boulevard Saint Michel, F-75005 Paris, France
\end{abstract}

Note: This paper is part of the JCP Emerging Investigators Special Collection.

a) Author to whom correspondence should be addressed: eric.bremond@u-paris.fr

\begin{abstract}
We recently derived a new and simple route to the determination of the range-separation parameter in range-separated exchange hybrid and double-hybrid density functionals by imposing an additional constraint to the exchange-correlation energy to recover the total energy of the hydrogen atom [Brémond et al., J. Chem. Phys. 15, 201102 (2019)]. Here, we thoroughly assess this choice by statistically comparing the derived values of the range-separation parameters to the ones obtained using the optimal tuning (OT) approach. We show that both approaches closely agree, thus, confirming the reliability of ours. We demonstrate that it provides very close performances in the computation of properties particularly prone to the one- and many-electron self-interaction errors (i.e., ionization potentials). Our approach arises as an alternative to the OT procedure, conserving the accuracy and efficiency of a standard Kohn-Sham approach to density-functional theory computation.
\end{abstract}

Published under license by AIP Publishing. https://doi.org/10.1063/5.0010976

\section{INTRODUCTION}

Within the Kohn-Sham approach to density-functional theory (KS-DFT), ${ }^{1,2}$ the concept of range separation relies on the splitting of the electron-electron interaction into short- and long-range (SR and LR, respectively) terms. ${ }^{3}$ Originally introduced by Savin at the end of the last century, it was conceived as a possible remedy to (partly) cure the recurrent errors in DFT, such as the one-electron self-interaction error (SIE) and its extension to many-electron systems. ${ }^{4,5}$ It is based on the observation that (semi)local approximations to the exchange-correlation energy accurately describe wavelength density fluctuations at short interelectronic distances but failed in the long-range regime. ${ }^{6,7}$ From there, the splitting of the Coulomb interaction has led over the years to a multitude of variants combining exchange-correlation (semi)local approximations in the short range to more sophisticated $a b$ initio wavefunction approaches in the long-range regime.

To a lesser extent, the concept of range separation gained popularity, thanks to its restriction to the treatment of the exchange energy only. ${ }^{14-16}$ The resulting "long-range corrected" or "Coulomb attenuated" approaches are considered as part of the hybrid class of approximations to the exchange-correlation energy. ${ }^{17}$ With respect to more standard global hybrids (GHs) such as the well-known ${\mathrm{B} 3 L Y \mathrm{P}^{18-20} \text { or PBE0 }}^{21,22}$ expressions, their fraction of exact-like exchange (EXX) is a function of the interelectronic distance so that the LR exchange interaction is fully, ${ }^{14,15}$ or just largely for a few of them, ${ }^{16}$ described by the nonlocal exchange. This switching between (semi)local and exact-like exchange terms physically imposes the recovery of the correct asymptote $-r_{12}^{-1}, r_{12}=\left|\mathbf{r}_{2}-\mathbf{r}_{2}\right|$ being the electron-electron distance, for the exchange potential. 
It, thus, corrects the density overdelocalization in the LR regime, which affects global hybrids when dealing with molecular properties such as the intra-/inter-molecular charge-transfer excitations, the dissociation of open-shell complexes, ${ }^{24-26}$ or the structure of poly- $\pi$-conjugated systems. ${ }^{27-29}$ Moreover, it conserves the systematic improvement offered by global hybrids with respect to semilocal approximations $^{30,31}$ and their successful modeling of a large number of ground- and excited-state properties. ${ }^{32,33}$

The hereby named range-separated exchange (RSX) scheme connects generally the SR to the LR regime through the standard error function. It allows not only a smooth connection between both regimes but also guarantees an analytic estimation of the bielectronic integrals in Gaussian basis set computations. ${ }^{15}$ By definition, this switching function depends on a parameter dubbed as the range-separation parameter, governing the interelectronic distance for which the switching between the SR and LR regions occurs. Its determination is, however, the topic of discussions. Originally, its value was systematically derived in an empirical fashion, that is to say, to minimize the errors computed on a training set of chemical properties. This approach is behind numerous wellknown RSX hybrid density functionals. A non-exhaustive list of some of them includes LC-BOP, ${ }^{14,15}$ CAM-B3LYP, ${ }^{16}$ LC- $\omega$ PBE, ${ }^{34-36}$ and, of course, the $\omega \mathrm{B} 97-$ and Minnesota-like families (e.g., see, for instance, Refs. 37-40 for the former and Refs. 41-44 for the latter).

In time, by dint of development, the empirical determination of the range-separation parameter evolved in a parameter-free fashion. In this way, Baer and co-workers developed the so-called tuned range-separated hybrid density functionals ${ }^{45}$ with the example of the BNL density functional. ${ }^{9,46}$ The determination of the rangeseparation parameter becomes in such a way system-dependent: its value is obtained according to an optimal tuning (OT) procedure to fulfill the Koopmans's theorem. ${ }^{47}$ The OT approach initially demonstrated its success with long-range through-space charge-transfer excitations occurring in molecular dimers ${ }^{48}$ and, in time, largely proved its robustness through numerous ground- and excited-state applications especially prone to one- and many-electron SIEs. ${ }^{49-54}$ However, even in its self-consistent fashion formulation (scOT), the approach remains prone to size-consistency-related issues and is of course computationally expensive. ${ }^{56}$

To remedy these issues, still keeping a nonempirical ambition, we recently proposed an alternative to the previous ways of determining the range-separation parameter. The approach consists in releasing the RSX hybrid density functional from the oneelectron SIE by setting it to reproduce the absolute energy of the simplest chemical system, i.e., the hydrogen atom. ${ }^{57,58}$ In this way, we transformed five nonempirical density functionals belonging to the pure, hybrid, and double-hybrid (DH) class of approximations, i.e., PBE, ${ }^{59} \mathrm{PBE} 0,{ }^{21,22} \mathrm{PBE} 0-1 / 3,{ }^{60} \mathrm{PBE} 0-\mathrm{DH},{ }^{61}$ and PBE-QIDH, into their RSX variants, all of them mixing different fractions of EXX in their SR and imposing a full EXX description to their LR asymptote. Their performances with respect to challenging molecular properties, especially prone to SIEs, were comparable, and sometimes better than other popular parameterized approaches, ${ }^{27,57,58}$ demonstrating, thus, the robustness of the approach despite its simplicity.

In this quest for the best determination of the range-separation parameter, we want to go here a step further by showing that our nonempirical (and cost-effective) way of determining the rangeseparation parameter is not only validated by benchmarking energy properties but it is also close, in terms of numerical results and parameterization, to the OT procedure. More precisely, we want to show that our approach provides a range-separation parameter statistically representative of the one obtained by OT and that the resulting computed properties between both approaches are in close agreement.

\section{THEORY}

The Jacob's Ladder sums up the five classes of exchangecorrelation density functional approximations (DFAs), all of them ranked according to their level of nonlocality. ${ }^{17}$ On rank 4, the global hybrid (GH) approximation hybridizes the exchange component of the density functional. It introduces a mixing of nonlocal EXX with a pure semilocal density functional approximation (DFA) such as

$$
E_{x c}^{\mathrm{GH}}=a_{x} E_{x}^{\mathrm{EXX}}+\left(1-a_{x}\right) E_{x}^{\mathrm{DFA}}+E_{c}^{\mathrm{DFA}},
$$

where $a_{x}$ governs the fraction of EXX, and $E_{x}^{\mathrm{DFA}}$ and $E_{c}^{\mathrm{DFA}}$ denote the semilocal exchange and correlation energy terms, respectively. $E_{x}^{\mathrm{DFA}}$ and $E_{c}^{\mathrm{DFA}}$ are generally expressed at a lower rank of approximation that can be the local density (rank 1), generalized gradient (rank 2), or meta-generalized gradient (rank 3) approximation (LDA, GGA, or meta-GGA, respectively).

The nonlocal character introduced to the exchange within the GH approximation can be extended to the correlation. The hybridization of the latter component occurs on rank 5 of the Jacob's Ladder. The resulting double-hybrid (DH) approximation is a generalization of Eq. (1). It introduces a fraction of nonlocal second-order perturbation theory (PT2) correlation such as ${ }^{63-65}$

$$
E_{x c}^{\mathrm{DH}}=a_{x} E_{x}^{\mathrm{EXX}}+\left(1-a_{x}\right) E_{x}^{\mathrm{DFA}}+a_{c} E_{c}^{\mathrm{PT} 2}+\left(1-a_{c}\right) E_{c}^{\mathrm{DFA}},
$$

where $a_{c}$ controls the fraction of PT2 correlation and $a_{x}$ still governs, as above, the fraction of EXX.

Both $\mathrm{GH}$ and $\mathrm{DH}$ approximations contribute themselves to (partly) cure some of the DFT drawbacks. As example, GHs provide an answer to the one-electron SIE and its many-electron extension by (partially) canceling the spurious interaction of an electron with itself. ${ }^{4,5}$ Among others, DHs add to the latter feature, ${ }^{30}$ a correction to the repulsive behavior of van der Waals dimers by avoiding the electron correlation to vanish at a large distance. ${ }^{66}$ All these significant improvements with respect to semilocal approximations are demonstrated by numerous benchmark investigations and applications dealing with energy and structure properties on ground and excited states. ${ }^{31,67-71}$ However, the wrong asymptotic description of their exchange potential, behaving as $-a_{x} \cdot r_{12}^{-1}$ instead of $-r_{12}^{-1}$, deteriorates the estimation of chemical properties especially prone to LR interactions.

The RSX scheme applied to the exchange potential allows us, however, to recover the correct asymptote in the LR regime on condition that a full fraction of EXX describes it. ${ }^{15}$ It consists in a physically sound solution splitting the Coulomb operator into SR and LR terms through the standard error function, 


$$
\frac{1}{r_{12}}=\underbrace{\frac{1-\left[\alpha+\beta \operatorname{erf}\left(\mu r_{12}\right)\right]}{r_{12}}}_{\mathrm{SR}=\text { short range }}+\underbrace{\frac{\alpha+\beta \operatorname{erf}\left(\mu r_{12}\right)}{r_{12}}}_{\text {LR=long range }},
$$

where $\alpha$ and $\alpha+\beta$ are two combined parameters governing the mixing of EXX in the SR and LR regimes, respectively, and $\mu$ is the range-separation parameter controlling the switching distance for which one term dominates the other. The application of the rangeseparation transformation to the exchange energy defines, thus, the range-separated exchange hybrid energy (RSX-H) such as

$$
E_{x, \mu}^{\mathrm{RSX}-\mathrm{H}}=\alpha E_{x}^{\mathrm{EXX}}+\beta E_{x, \mu}^{\mathrm{EXX}}+(1-\alpha) E_{x}^{\mathrm{DFA}}-\beta E_{x, \mu}^{\mathrm{DFA}} .
$$

According to Eq. (3), the recovery of the expected $-r_{12}^{-1}$ asymptote for the exchange potential in the LR regime imposes to both $\alpha$ and $\beta$ parameters to first verify the relation $\alpha+\beta=1$. Moreover, according to Eq. (4), the conservation of the EXX and DFA mixing in the SR regime set by GH or DH expressions [see Eq. (1) or (2)] imposes $\alpha$ to be equal to $a_{x}$. In this context, the application of the RSX scheme to the exchange-correlation DH energy expression provided by Eq. (2) finally gives

$$
E_{x c, \mu}^{\mathrm{RSX}-\mathrm{DH}}=E_{x c}^{\mathrm{DH}}+\left(1-a_{x}\right) E_{x, \mu}^{\mathrm{EXX}}-\left(1-a_{x}\right) E_{x, \mu}^{\mathrm{DFA}} .
$$

It is of note to recall that the $\mathrm{DH}$ approximation is a generalization of the GH one; thus, the exchange-correlation RSX-H energy expression is recovered by setting $a_{c}=0$ in Eq. (5). From the latter equation, all pure, $\mathrm{GH}$, and DH density functionals can, thus, be transformed into their RSX variants. In a recent investigation, ${ }^{57,58}$ we, indeed, applied this transformation to a nonempirical family of pure (i.e., PBE), GH (i.e., PBE0 and PBE0-1/3), and $\mathrm{DH}$ (i.e., PBE0-DH and PBE-QIDH) density functionals whose parameters are defined in Table I. The introduced range-separation parameter $\mu$ was derived in a nonempirical fashion to reproduce the absolute energy of the hydrogen atom as said before.

In the present work, we want to verify that the derived rangeseparation parameter is statistically significant and remains in line with the OT procedure. ${ }^{45}$ The latter approach consists in the tuning of the $\mu$ value entering into the RSX-H density functional in order to force the Koopmans's theorem,

$$
-\varepsilon_{\mathrm{HOMO}}^{\mathrm{RSX}-\mathrm{H}}(N, \mu)=E^{\mathrm{RSX}-\mathrm{H}}(N-1, \mu)-E^{\mathrm{RSX}-\mathrm{H}}(N, \mu),
$$

with $N$ the number of electrons of the molecular system under investigation, $\varepsilon_{\mathrm{HOMO}}^{\mathrm{RSX}-\mathrm{H}}$ the ground-state eigenvalue of the highestoccupied molecular orbital (HOMO), and $E^{\mathrm{RSX}-\mathrm{H}}$ the ground-state

TABLE I. Fraction of exact-like exchange $a_{x}$, range-separation parameter $\mu\left(\right.$ bohr $\left.^{-1}\right)$, and fraction of second-order perturbation theory correlation $a_{c}$ defining the exchangecorrelation density functionals derived from Eq. (5) and developed in Ref. 57.

\begin{tabular}{lccc}
\hline \hline DF & $a_{x}$ & $\mu$ & $a_{c}$ \\
\hline RSX-PBE & 0 & 0.45 & 0 \\
RSX-PBE0 & $1 / 4$ & 0.39 & 0 \\
RSX-PBE0-1/3 & $1 / 3$ & 0.37 & 0 \\
RSX-0DH & $1 / 2$ & 0.33 & $1 / 2^{3}$ \\
RSX-QIDH & $3^{-1 / 3}$ & 0.27 & $1 / 3$ \\
\hline \hline
\end{tabular}

self-consistent field (SCF) energy of the considered ionized state. The right member of Eq. (6) is equal to the vertical ionization potential (IP) of the molecular system computed according to the $\triangle \mathrm{SCF}$ approach (it will be shortly, thus, referred to as $\Delta_{\mathrm{IP}} E^{\mathrm{RSX}-\mathrm{H}}$ in the following).

The Koopmans's theorem is, however, restricted to SCF-based approaches, providing a total energy minimized with respect to the total set of orbitals. DH density functionals, which include a fraction of PT2 correlation energy computed a posteriori from a set of self-consistently obtained KS orbitals, are, by definition, out of this framework and belong to the extended variant of the Koopmans's theorem ${ }^{72,73}$ and its application to PT2-like approaches. ${ }^{74,7}$ It adds to the ground-state eigenvalue of the HOMO, the relaxation of the PT2 component via the first- and second-order reduced density matrices. The OT procedure applied to the RSX-DH density functional becomes

$$
-\varepsilon_{\mathrm{HOMO}}^{\mathrm{RSX}-\mathrm{DH}}(N, \mu)=E^{\mathrm{RSX}-\mathrm{DH}}(N-1, \mu)-E^{\mathrm{RSX}-\mathrm{DH}}(N, \mu),
$$

with $E^{\mathrm{RSX}-\mathrm{DH}}$ the ground-state total PT2 energy of the considered ionized state and $\varepsilon_{\mathrm{HOMO}}^{\mathrm{RSX}-\mathrm{DH}}$ the corresponding relaxed RSX-DH eigenvalue of the HOMO. At this early stage, we are unfortunately unable to propose an implementation leading to the exact computation of $\varepsilon_{\mathrm{HOMO}}^{\mathrm{RSX}-\mathrm{DH}}$. In a first approximation, we will, thus, neglect the $\mathrm{PT} 2$ relaxation component to the orbital energy and impose $\varepsilon_{\mathrm{HOMO}}^{\mathrm{RSX}-\mathrm{DH}} \approx \varepsilon_{\mathrm{HOMO}}^{\mathrm{RSX}-\mathrm{H}}$ during the OT procedure for the RSX-DH density functional. However, it is of note to recall that within the second-order MøllerPlesset (MP2) theory framework, some recent investigations go a step further and show the importance of this component during the direct estimation of ionization potential properties. ${ }^{74,7}$

\section{COMPUTATIONAL DETAILS}

All the computations are performed with the release B.01 of the Gaussian' 16 program. ${ }^{77}$ Each single point energy calculation is performed using a tight SCF convergence criteria and an ultrafine integration grid. For the RSX-PBE, RSX-PBE0, and RSX-PBE0-1/3 range-separated exchange hybrid (RSX-H) density functionals, the optimal tuning (OT) procedure is performed by successive energy single point evaluations at a fixed molecular structure, making the range-separation parameter $\mu$ to vary up to the equalization of the first ionization potential of the $N$-electron system (computed according to a $\triangle \mathrm{SCF}$ procedure) to the opposite energy value of its HOMO orbital [see Eq. (6)]. The resulting tuned range-separation parameter is denoted as $\mu^{\mathrm{OT}}$ and is expressed in $\mathrm{bohr}^{-1}$. The application of the OT procedure to the RSX-0DH and RSX-QIDH doublehybrid (RSX-DH) density functionals follows the same protocol except that the first ionization potential of the $N$-electron system is computed according to a $\triangle \mathrm{PT} 2$ procedure [see Eq. (7)]. Note that the RSX-0DH(SCF) and RSX-QIDH(SCF) density functionals belong to the RSX-H model and are estimated as the SCF energy contribution of the RSX-0DH and RSX-QIDH density functionals, i.e., by neglecting the addition of the PT2 contribution.

Unless otherwise noted, the computations are performed with the very large def2-QZVP Ahlrichs's quadruple- $\zeta$ basis set, ${ }^{78}$ which assures a nearly complete basis set convergence and minimizes the basis set superposition error. To keep a consistency with our previous investigation, ${ }^{57}$ the OT procedure is applied at this level 
of theory to the 100 molecular systems belonging to the GW100 database, ${ }^{79}$ which gathers 100 reference IP energies computed with the coupled-cluster singles and doubles plus perturbative triples $[\mathrm{CCSD}(\mathrm{T})]$ approach. ${ }^{80}$ To complement this investigation, the performances of the pristine RSX-H and RSX-DH density functionals ${ }^{5}$ are compared to the OT ones for system properties especially prone to the one- and many-electron SIEs that are the IP of helium clusters ${ }^{81}$ and the dissociation of the $\mathrm{NaCl}$ molecule. ${ }^{36}$ On these specific examples, the energy properties are computed by adding diffuse $s$ and $p$ functions from aug-cc-pVQZ ${ }^{82}$ to def2-QZVP, thus, giving the aug-def2-QZVP basis set.

\section{RESULTS AND DISCUSSION}

The GW100 database is an extended set of 100 structures of small organic molecules built to probe the first IP energy property. ${ }^{79}$ Owing to its large size and broad variety of systems, it provides a fair statistical survey of the performance of any computational approach with respect to this property. Moreover, it counts for 100 reference first IP energies computed at the "gold standard" $\operatorname{CCSD}(\mathrm{T})$ level of theory, ${ }^{80}$ a considerable advantage for benchmarking purpose since it avoids the possible bias introduced by experimental references. In a recent investigation, ${ }^{57}$ we took advantage of the quality of this database to test the performance of 5 newly developed nonempirical RSX-H and RSX-DH density functionals, whose range-separation parameters were determined to recover the absolute total energy of the hydrogen atom (Table I). We demonstrated that on this database, the resulting RSX approaches provided IP estimates with mean absolute deviations (MADs) lying around $\sim 0.2 \mathrm{eV}$. Here, we start by comparing the pristine range-separation parameter $\mu$ (determined in a nonempirical fashion) to the statistical ensemble provided by the optimally tuned variant of each density functional on the GW100 database. Figure 1 reports this analysis for the density functionals belonging to the RSX-H class of approximations, i.e., RSX-PBE0 and RSX-PBE0-1/3, together with RSX-PBE. For each approach, the histogram distribution of the optimally tuned range-separation parameter $\left(\mu^{\mathrm{OT}}\right)$ is relatively narrow. The standard deviations are, indeed, estimated to be $0.21 \mathrm{bohr}^{-1}, 0.25 \mathrm{bohr}^{-1}$, and $0.25 \mathrm{bohr}^{-1}$ for RSX-PBE, RSX-PBE0, and RSX-PBE0-1/3, respectively (Table II). Each histogram distribution is centered on a value, in average, in close agreement with $\mu$, despite the presence of some outlier systems such as the phosphorus dimer $\left(\mu^{\mathrm{OT}}=2.05\right.$ bohr $^{-1}$ with RSX-PBE). More details about the outlier systems are provided in Table SI within the supplementary material. The mean values are found as $0.42 \mathrm{bohr}^{-1}, 0.35 \mathrm{bohr}^{-1}$, and 0.32 bohr $^{-1}$ for RSX-PBE, RSX-PBE0, and RSX-PBE0-1/3, respectively, in comparison to the pristine values of $\mu$, which are $0.45 \mathrm{bohr}^{-1}, 0.39 \mathrm{bohr}^{-1}$, and $0.37 \mathrm{bohr}^{-1}$, respectively. The deviations obtained between both approaches are very small, i.e., lower than $0.05 \mathrm{bohr}^{-1}$. It confirms de facto the trend found for our nonempirical approach.

By definition, the OT procedure enforces the recovery of the Koopmans's theorem. As a result, the correlation between the IP energy computed according to a $\triangle \mathrm{SCF}$ approach $\left(\Delta_{\mathrm{IP}} E\right)$ and the opposite value of the HOMO orbital energy $\left(-\varepsilon_{\mathrm{HOMO}}\right)$ is another indicator of the quality of the method. Figure 1 depicts those correlations for the pristine (black circles) and optimally tuned variants (blue circles) of each RSX-H density functional. The correlations of the latter are of course optimal with a $R^{2}=1.00$ coefficient for each density functional imposed by definition. For the former ones, the correlation is also very close to $R^{2}$ coefficients larger than 0.97 , proving once again the reliability of our minimally empirical approach.

The same statistical treatment is then applied to the RSX-0DH and RSX-QIDH density functionals, which belong to the DH class of approximations. As recalled by Eq. (2), DHs are composed by a SCF exchange-correlation energy part, which once converged, is used to compute an a posteriori PT2 correlation energy part. We first examine through Fig. 2 the statistical analysis of the range-separation parameter on the GW100 database for the SCF part of each RSX$\mathrm{DH}$, dubbed as RSX-0DH (SCF) and RSX-QIDH (SCF), just as the ones of the full RSX-DHs. For the former ones, the OT procedure allows strict fulfilment of the Koopmans's theorem since they belong

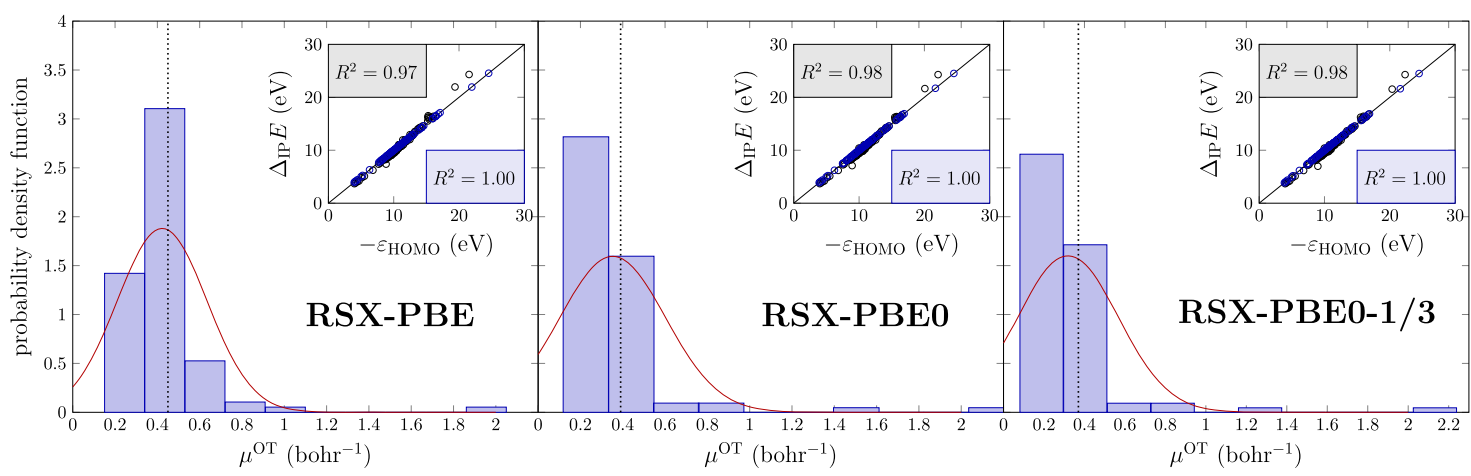

FIG. 1. Probability density function of the range-separation parameter $\mu^{\text {OT }}\left(\right.$ bohr $\left.^{-1}\right)$ determined from the computation of the ionization potential (IP) energies of the 100 small molecules gathered into the GW100 dataset following the optimal tuning (OT) procedure. The black dashed vertical line sets the pristine $\mu$ value of each range-separated hybrid density functional. The inset correlation diagrams compare the IP energies computed as the energy difference between the cationic and the neutral system $\Delta_{\mathrm{IP}} E$, and the energy of the HOMO orbital $\varepsilon_{\text {HOMO }}$. Blue dots and the corresponding $R^{2}$ coefficients of determination derive from the OT procedure, while black ones are obtained with the corresponding pristine range-separated hybrid density functional. All computations are performed with the def2-QZVP basis set. 
TABLE II. Mean absolute deviations calculated over the ionization potential energies (eV) of the 100 small molecules gathered into the GW100 dataset computed as the energy difference between the cationic and the neutral system $\Delta_{\mathrm{IP}} E$, and the energy of the HOMO orbital $\varepsilon_{\text {HOMO }}$. The mean optimally tuned range-separation parameters $\bar{\mu}^{\mathrm{OT}}$ (bohr ${ }^{-1}$ ) and the standard deviations $\sigma_{\mu}$ От $\left(\right.$ bohr $\left.^{-1}\right)$ are also provided for the OT-based approaches. The pristine $\mu\left(\mathrm{bohr}^{-1}\right)$ values are provided for comparison. All computations are performed with the def2-QZVP basis set. The reference values are taken from Ref. 80.

\begin{tabular}{|c|c|c|c|c|c|c|c|}
\hline & RSX-PBE & RSX-PBE0 & RSX-PBE0-1/3 & RSX-0DH(SCF) & RSX-0DH & RSX-QIDH(SCF) & RSX-QIDH \\
\hline \multicolumn{8}{|c|}{ Pristine } \\
\hline$\Delta_{\mathrm{IP}} E$ & 0.27 & 0.25 & 0.25 & 0.27 & 0.25 & 0.42 & 0.24 \\
\hline$-\varepsilon_{\text {HOMO }}$ & 0.46 & 0.47 & 0.50 & 0.49 & 0.49 & 0.49 & 0.49 \\
\hline$\mu^{\mathrm{a}}$ & 0.45 & 0.39 & 0.37 & 0.33 & 0.33 & 0.27 & 0.27 \\
\hline \multicolumn{8}{|c|}{ Optimal tuning } \\
\hline$\Delta_{\mathrm{IP}} E$ & 0.28 & 0.22 & 0.20 & 0.26 & 0.18 & 0.44 & 0.20 \\
\hline$-\varepsilon_{\text {HOMO }}$ & 0.28 & 0.22 & 0.20 & 0.24 & 0.18 & 0.38 & 0.21 \\
\hline $\bar{\mu}^{\mathrm{OT}}$ & 0.42 & 0.35 & 0.32 & 0.24 & 0.27 & 0.15 & 0.24 \\
\hline$\sigma_{\mu} \mathrm{OT}$ & 0.21 & 0.25 & 0.25 & 0.26 & 0.26 & 0.37 & 0.36 \\
\hline
\end{tabular}

${ }^{\mathrm{a}}$ From Refs. 57 and 58.

to the RSX-H class of approximations. The standard deviations of the $\mu^{\mathrm{OT}}$ ensembles are in line for RSX-0DH (SCF) $\left(0.27 \mathrm{bohr}^{-1}\right)$ or slightly larger for RSX-QIDH (SCF) $\left(0.37 \mathrm{bohr}^{-1}\right)$, in comparison to the previous RSX-H density functionals (Table II). The mean values are $0.24 \mathrm{bohr}^{-1}$ and $0.15 \mathrm{bohr}^{-1}$, respectively, in comparison to those of the pristine $\mu$, which are $0.33 \mathrm{bohr}^{-1}$ and $0.27 \mathrm{bohr}^{-1}$, respectively. As a result, the deviations between the mean values of $\mu^{\mathrm{OT}}$ and those of the pristine $\mu$ are not larger than $0.12 \mathrm{bohr}^{-1}$, but are, indeed, larger that the agreement found for RSX-PBE, RSX-PBE0, and RSX-PBE0-1/3. Going now to the full RSX-DH

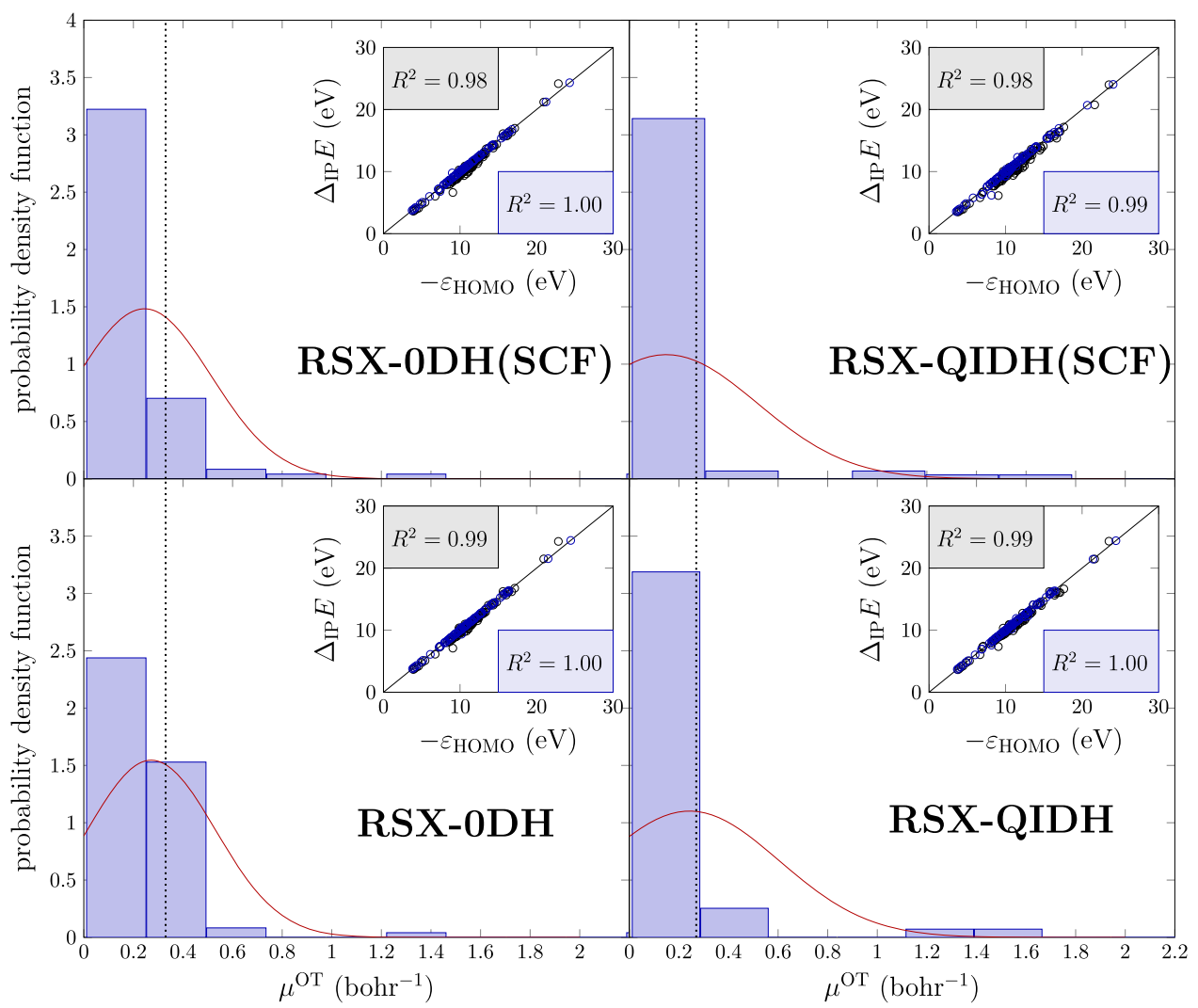

FIG. 2. Probability density function of the range-separation parameter $\mu^{\text {OT }}$ $\left(b{ }^{-1}\right)$ determined from the computation of the ionization potential (IP) energies of the 100 small molecules gathered into the GW100 dataset following the optimal tuning (OT) procedure. The black dashed vertical line sets the pristine $\mu$ value of each range-separated doublehybrid density functional. The inset correlation diagrams compare the IP energies computed as the energy difference between the cationic and the neutral system $\Delta_{\mathrm{IP}} E$, and the energy of $\mathrm{HOMO}$ orbital $\varepsilon_{\text {HOMO }}$. Blue dots and the corresponding $R^{2}$ coefficients of determination derive from the OT procedure, while black ones are obtained with the corresponding pristine range-separated hybrid density functional. All computations are performed with the def2-QZVP basis set. 


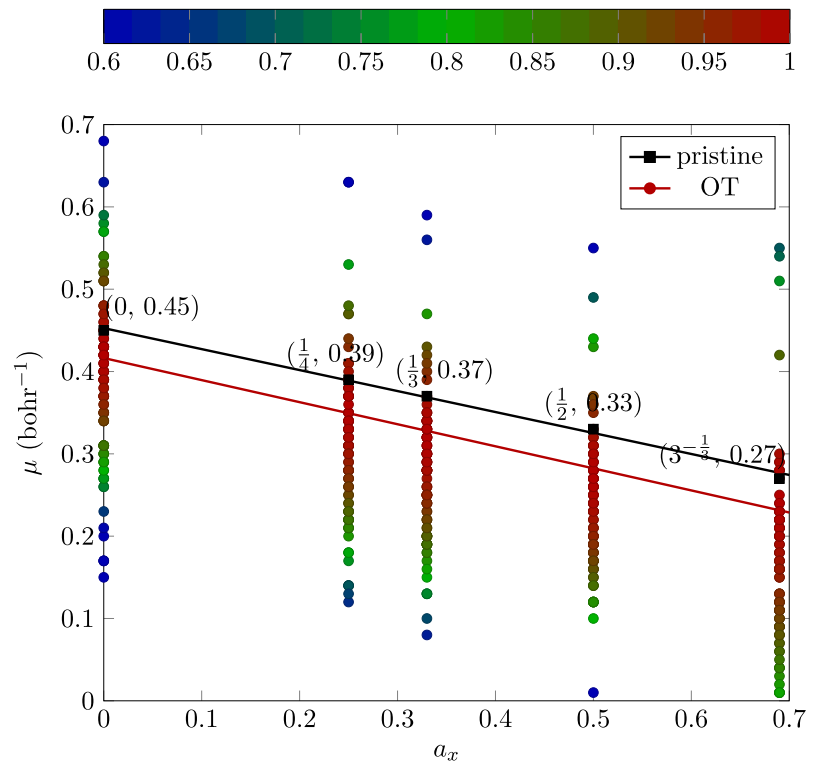

FIG. 3. (Black) Pristine and (colored) optimally tuned range-separation parameters ( $\mu$ and $\mu^{\mathrm{OT}}$, respectively, in bohr ${ }^{-1}$ ) as functions of the whole fraction of exactlike exchange $a_{x}$. The color map follows a normalized Gaussian distribution and depicts the density of $\mu^{\text {OT }}$ for each value of $a_{x}$. For each collection of points, a linear regression (solid line) is performed such as $\mu=-0.25 a_{x}+0.45$ and $\mu^{\text {OT }}$ $=-0.27 a_{x}+0.41$, respectively.

density functionals, approximating the extended Koopmans's theorem improves the statistical agreement between the mean values of $\mu^{\mathrm{OT}}$ and those of the pristine $\mu$. They are here found to be 0.27 bohr ${ }^{-1}$ and 0.24 bohr $^{-1}$ for RSX-0DH and RSX-QIDH, respectively, leading, thus, to deviations not larger than $0.05^{-1}$. The correlation diagrams between the IP energy computed according to a $\triangle \mathrm{PT} 2$ approach and $-\varepsilon_{\text {HOMO }}$ confirm the excellent agreement between the pristine and OT variants of the two RSX-DHs, exhibiting $R^{2}$ coefficients of about 0.99 and 1.00, respectively (Fig. 2).
Above all, the agreement found between the mean values of $\mu^{\text {OT }}$ and those of the pristine $\mu$ also reproduces the decreasing trend as a function of the fraction $a_{x}$ of EXX (Fig. 3). The linear relationship observed in the domain $a_{x} \in\left[0,3^{-1 / 3}\right]$ is difficult to rationalize and can, indeed, be viewed as a rule of thumb. However, both approaches faithfully reproduce it with a slope of about -0.25 a.u. for the pristine density functionals and -0.27 a.u. for the optimally tuned ones. This decreasing trend indicates that for both approaches, the larger the $a_{x}$, the slower the switch from the SR semilocal term to the LR EXX one has to be. It, thus, underlines the importance of the semilocal term of the density functional, especially when $a_{x}$ is large.

The statistical agreement between the pristine and OT variants is not only found during the determination of the range-separation parameter but also during the computation of the first IP energy property. Table II reports the corresponding MADs associated with the GW100 database when IPs are estimated through the $\Delta_{\mathrm{IP}} E$ or $-\varepsilon_{\text {Hомо }}$ energy values for the set of investigated density functionals. For the OT-based methods, it is important to note the perfect agreement between both energy criteria. However, RSX-0DH(SCF) and RSX-QIDH(SCF) slightly deviate from this trend due to the numerical errors caused by the $10^{-2}$ bohr $^{-1}$ threshold we set for $\mu$ while enforcing the recovery of the Koopman's theorem. These deviations emphasize, thus, how difficult and time-consuming the OT procedure is. With respect to the $\Delta_{\mathrm{IP}} E$ measure, the disagreement between the pristine and OT variants is not larger than $0.07 \mathrm{eV}$. It is, however, larger for the $-\varepsilon_{\text {HOMO }}$ measure and exceeds $0.2 \mathrm{eV}$ given that only the OT procedure recovers the Koopmans's theorem. Out of that, the RSX-QIDH and RSX-0DH DHs are found as the most accurate approaches within the pristine and OT variants, respectively, with MADs equal to $0.24 \mathrm{eV}$ and $0.18 \mathrm{eV}$, respectively. The best performing RSX-H is RSX-PBE0-1/3 with an MAD equal to $0.25 \mathrm{eV}$ with the pristine $\mu$ value and $0.20 \mathrm{eV}$ with the OT one.

As a further validation test, we select the helium cluster system of size $n\left(\mathrm{He}_{n}\right)$, which is demonstrated as an excellent test case to measure the delocalization error (DE) of a density functional. ${ }^{81}$ This error is readily calculated as the energy difference between the first IP energy of a cluster of size $n=16$, whose size is assumed to be large enough to provide a converged IP energy, and $n=1$. In

TABLE III. Ionization potential (eV) of helium clusters of size $n$ computed with the pristine and optimally tuned range-separated exchange hybrid and double hybrids considered in this investigation at the aug-def2-QZVP level of theory. The delocalization error $(\mathrm{eV})$ is calculated as $D E=I P\left(\mathrm{He}_{1}\right)-\mathrm{IP}\left(\mathrm{He}_{16}\right)$. The optimally tuned range-separation parameter $\mu^{\mathrm{OT}}\left(\mathrm{bohr}^{-1}\right)$ is given in parenthesis. The reference value is taken from Ref. 81.

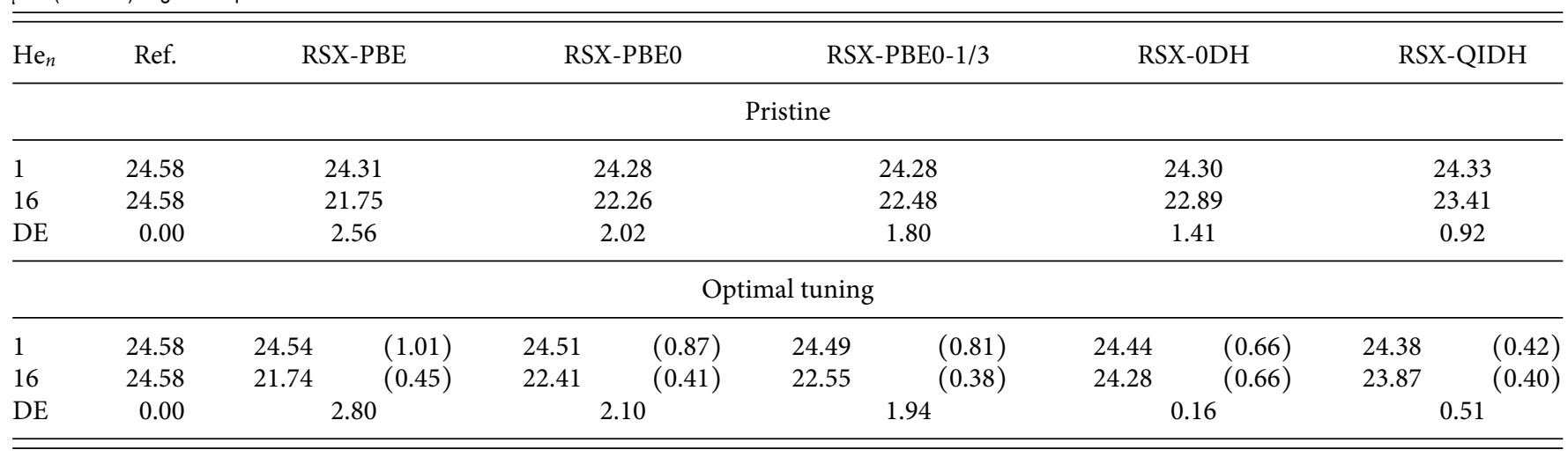




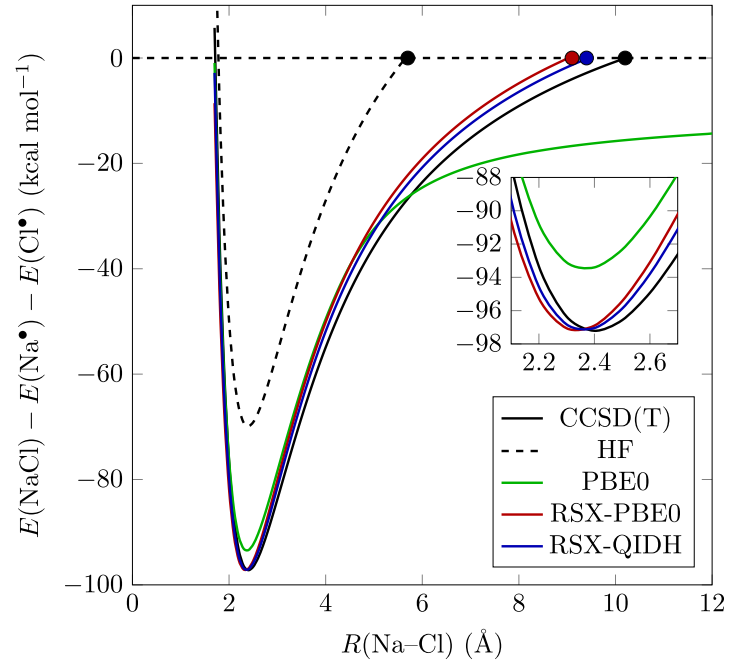

FIG. 4. Dissociation energy profile of the $\mathrm{NaCl}$ molecule $\left(\mathrm{kcal} \mathrm{mol}^{-1}\right)$ with respect to $E\left(\mathrm{Na}^{*}\right)+E\left(\mathrm{Cl}^{*}\right)$ computed with the aug-def2-QZVP basis set. Bullets set the critical separation distance $R_{c}(\AA)$ to be compared with the experimental one $R_{c}$ $=9.4 \AA$ taken from Ref. 36. The inset is a zoomed-in image of the binding region.

the OT formulation, the range-separation parameter of the RSX-H density functionals is system-dependent (Table III). Indeed, its value decreases when the size of the cluster increases. As an example, it goes from 1.01 when $n=1 \mathrm{bohr}^{-1}$ to $0.45 \mathrm{bohr}^{-1}$ when $n=16$ for the RSX-PBE density functional. This trend shows that the switching between the SR and LR regimes has to occur at a shorter interelectronic distance for very small systems than that for larger systems to fulfill the Koopmans's theorem. It, thus, confirms the sizeconsistency-related issues already underlined by Karolewski and co-workers in Ref. 56.
Regarding the DE by itself, the pristine variants of the RSX$\mathrm{H}$ density functionals perform better than the OT ones (Table III). Their DEs are found lower by more than $\sim 0.1 \mathrm{eV}$ with respect to the pristine than OT approaches. The opposite trend is, however, observed for the RSX-DH density functionals, which do not exactly fulfill the Koopmans's theorem. As an example, the DE goes from 2.80 (OT) to 2.56 (pristine) $\mathrm{eV}$ in the case of RSX-PBE and from 0.51 (OT) to 0.92 (pristine) eV for RSX-QIDH. It also shows that the more a RSX-H mixes a high fraction of EXX in its SR (RSX$\mathrm{PBE}, \mathrm{EXX}=0 \%$ vs RSX-PBE0-1/3, and $\mathrm{EXX}=\sim 33 \%$ ), the more its $\mathrm{DE}$ decreases, and that the RSX-DH approximation is found as less prone to the DE than the RSX-H, the DE of RSX-PBE being $2.56 \mathrm{eV}$, while the one of RSX-QIDH being $0.92 \mathrm{eV}$ in the case of the pristine approach.

The last validation example is the dissociation of the $\mathrm{NaCl}$ molecule in its neutral $\mathrm{Na}^{\bullet}$ and $\mathrm{Cl}^{\bullet}$ atoms. The system is of particular interest to force the density functionals to test the SIE since the ionization state of each atom changes along the dissociation path. ${ }^{36,83,84}$ In the binding region, both atoms make an ionic pair which persists up to a critical distance, dubbed as $R_{c}$, for which a charge transfer occurs, imposing a dissociation limit made by neutral $\mathrm{Na}^{\bullet}$ and $\mathrm{Cl}^{\bullet}$ atoms. ${ }^{85}$ Semilocal or global hybrid density functionals mixing a small fraction of EXX fail to describe this charge transfer and keep an anionic pair with a spurious fractional charge and a strong interaction energy lowering at the dissociation limit (Fig. 4). However, their transformation into a RSX-H functional overcomes this failure. The pristine RSX-H and RSX-DH approximations recover the reference experimental and $\operatorname{CCSD}(\mathrm{T})$ features with a correct dissociation limit in neutral $\mathrm{Na}^{\bullet}$ and $\mathrm{Cl}^{\bullet}$ atoms (Table IV). The binding energy is relatively well reproduced with respect to the experimental reference $\left(-98.2 \mathrm{kcal} \mathrm{mol}^{-1}\right)$, the best density functional being RSX-PBE with an overbinding energy of about $0.1 \mathrm{kcal} \mathrm{mol}^{-1}$. With respect to $\operatorname{CCSD}(\mathrm{T})$, which estimates the binding energy to be $-97.2 \mathrm{kcal} \mathrm{mol}^{-1}$, that is, $+1.0 \mathrm{kcal} \mathrm{mol}^{-1}$ larger than the experimental value; the best RSX-H (RSX-DH) density

TABLE IV. Binding energy $\left(E_{\text {bind }}\right.$ in $\left.\mathrm{kcal} \mathrm{mol}^{-1}\right)$, critical separation distance $\left(R_{\mathrm{c}}\right.$ in $\left.\AA\right)$, and energy and Mulliken charge dissociation limits $\left(E_{\text {lim }}\right.$ in $\mathrm{kcal} \mathrm{mol}{ }^{-1}$ and $q_{\text {lim }}$ in a.u., respectively) of $\mathrm{NaCl}$ computed with the pristine and optimally tuned range-separated exchange hybrid and double hybrids considered in this investigation at the aug-def2-QZVP level of theory. The optimally tuned range-separation parameter derived at the binding distance $\mu_{\text {bind }}^{\mathrm{OT}}\left(\mathrm{bohr}^{-1}\right)$ is provided for OT-based approaches. The experimental reference is taken from Ref. 36 .

\begin{tabular}{|c|c|c|c|c|c|c|c|}
\hline $\mathrm{Na}-\mathrm{Cl}$ & Expt. & $\operatorname{CCSD}(\mathrm{T})$ & RSX-PBE & RSX-PBE0 & RSX-PBE0-1/3 & RSX-0DH & RSX-QIDH \\
\hline \multicolumn{8}{|c|}{ Pristine } \\
\hline$E_{\text {bind }}$ & -98.2 & -97.2 & -98.3 & -97.1 & -96.6 & -96.6 & -97.0 \\
\hline$R_{c}$ & 9.4 & 10.3 & 9.3 & 9.1 & 8.9 & 9.1 & 9.4 \\
\hline$E_{\lim }$ & $\ldots$ & 0.0 & 0.0 & 0.0 & 0.0 & 0.0 & 0.0 \\
\hline$q_{\lim }$ & $\ldots$ & $\ldots$ & 0.0 & 0.0 & 0.0 & 0.0 & 0.0 \\
\hline \multicolumn{8}{|c|}{ Optimal tuning } \\
\hline$\mu_{\text {bind }}^{\mathrm{OT}}$ & $\ldots$ & $\ldots$ & 0.40 & 0.32 & 0.32 & 0.23 & 0.15 \\
\hline$E_{\text {bind }}$ & -98.2 & -97.2 & -98.4 & -97.0 & -96.6 & -96.4 & -97.0 \\
\hline$R_{c}$ & 9.4 & 10.3 & 9.3 & 9.0 & 8.9 & 9.0 & 9.4 \\
\hline$E_{\lim }$ & $\ldots$ & 0.0 & 0.0 & 0.0 & 0.0 & 0.0 & 0.0 \\
\hline$q_{\lim }$ & $\ldots$ & $\ldots$ & 0.0 & 0.0 & 0.0 & 0.0 & 0.0 \\
\hline
\end{tabular}


functional is RSX-PBE0 (RSX-QIDH) with a binding energy of about $-97.1(-97.0) \mathrm{kcal} \mathrm{mol}^{-1}$. As for the critical distance, the best RSX$\mathrm{H}$ (RSX-DH) density functional with respect to experiment $\left(R_{c}=9.4\right.$ $\AA$ ) is RSX-PBE (RSX-QIDH) with an error of about $0.1(0.0) \AA$.

The OT procedure is difficult to set on a dissociation example such as this one because of its related size-consistency issue. ${ }^{56}$ However, by restricting its application to the minimum energy position, we find that the optimally tuned range-separation parameter $\mu_{\text {bind }}^{\mathrm{OT}}$ is again in agreement with the pristine one for each density functional. For the assessed series of RSX-H and RSX-DH density functionals, the trend is confirmed again, and its value decreases when the global fraction of EXX $a_{x}$ increases. Moreover, its value is in line with the pristine approach. As an example, $\mu_{\text {bind }}^{\mathrm{OT}}$ is tuned as $0.40 \mathrm{bohr}^{-1}$ for RSX-PBE, while it is equal to $0.45 \mathrm{bohr}^{-1}$ in the pristine approach and to $\mu_{\text {bind }}=0.15 \mathrm{bohr}^{-1}$ for RSX-QIDH, while $\mu=0.27$ bohr $^{-1}$. With these $\mu_{\text {bind }}$ values, the asymptotic behavior of each density functional faithfully recovers the dissociation of $\mathrm{NaCl}$ into its neutral atoms, with critical distance values in close agreement with the pristine approaches, the larger deviation being about $0.1 \AA ̊$.

\section{CONCLUSION}

The quest for the determination of the range-separation parameter has triggered a high number of studies focusing mostly on the optimization of its value (and other built-in parameters) with respect to large energy databases. Despite the robustness of the approach depicted by the large success of the $\omega \mathrm{B} 97^{37-40}$ or Minessota ${ }^{41-44}$ families of density functionals, we recently devoted our research to the nonempirical determination of the range-separation parameter. ${ }^{57,58}$ By tuning its value to recover the absolute total energy of the hydrogen atom, we built an entire family of range-separated exchange hybrid and double-hybrid density functionals mixing different fractions of EXX in their short range, while keeping a correct EXX long-range asymptotic behavior.

The choice of the hydrogen atom as a "physical condition" to tune the range-separation parameter was motivated as it is the simplest system for which we know exactly the absolute total energy. However, the performance and parameterization of the derived set of pristine density functionals with respect to other nonempirical approaches were still questionable. To confirm the previously derived values for the range-separation parameter, we here statistically compare them with the ones derived from the optimal tuning procedure applied to the very large GW100 molecular database. The resulting distribution of $\mu$ values is found relatively narrow and centered on mean values, which are in very close agreement with the ones belonging to the pristine approach, with deviations lower than $0.05 \mathrm{bohr}^{-1}$. For each range-separated hybrid or double-hybrid density functionals, the statistical proximity found for the range-separation parameters provides similar performances with respect to the computation of difficult properties especially prone to the one- and many-electron self-interaction errors, such as the first vertical IP energies estimated through the $\triangle \mathrm{SCF}$ or $\triangle \mathrm{PT} 2$ approaches.

With respect to the optimal tuning approach, ours is systemindependent. It results in a faster assessment of the targeted property in terms of computational cost without size-consistency-related issues, which, thus, allows a direct comparison of the system in its equilibrium and nonequilibrium structures, but leads to larger errors when properties such as IP energies are estimated through the opposite value of the HOMO energy since it does not strictly fulfill the Koopmans's theorem. Our nonempirical approach to determine the range-separated parameter can, thus, be viewed as an interesting compromise to the optimal tuning procedure, conserving the accuracy and efficiency of a standard KS-DFT computation, while partly recovering the interesting features of the optimal tuning procedure.

\section{SUPPLEMENTARY MATERIAL}

See the supplementary material for details about the density functional performances investigated herein.

\section{ACKNOWLEDGMENTS}

E.B. thanks ANR (Agence Nationale de la Recherche) and CGI (Commissariat à l'Investissement d'Avenir) for their financial support to this work through Labex SEAM (Science and Engineering for Advanced Materials and devices), Grant Nos. ANR-10-LABX096 and ANR-18-IDEX-0001. The authors thank the GENCI-CINES for HPC resources (Project Nos. A0040810359 and A0060810359). J.C.S.G. and A.J.P.J. thank the Ministerio de Ciencia e Innovación (Project No. PID2019-106114GB-I00).

\section{DATA AVAILABILITY}

The data that support the findings of this study are available within this article and its supplementary material.

\section{REFERENCES}

${ }^{1}$ W. Kohn and L. J. Sham, Phys. Rev. 140, A1133 (1965).

${ }^{2}$ P. Hohenberg and W. Kohn, Phys. Rev. 136, B864 (1964).

${ }^{3}$ A. Savin, in Recent Developments and Applications of Modern Density Functional Theory, edited by J. M. Seminario (Elsevier, Amsterdam, 1996), pp. 327-357.

${ }^{4}$ J. L. Bao, L. Gagliardi, and D. G. Truhlar, J. Phys. Chem. Lett. 9, 2353 (2018).

${ }^{5}$ A. J. Cohen, P. Mori-Sánchez, and W. Yang, Science 321, 792 (2008).

${ }^{6}$ K. Burke, J. P. Perdew, and D. C. Langreth, Phys. Rev. Lett. 73, 1283 (1994).

${ }^{7}$ D. C. Langreth and J. P. Perdew, Phys. Rev. B 15, 2884 (1977).

${ }^{8}$ I. Y. Zhang and X. Xu, J. Phys. Chem. Lett. 4, 1669 (2013).

${ }^{9}$ R. Baer and D. Neuhauser, Phys. Rev. Lett. 94, 043002 (2005).

${ }^{10}$ J. G. Ángyán, I. C. Gerber, A. Savin, and J. Toulouse, Phys. Rev. A 72, 012510 (2005).

${ }^{11}$ J. Toulouse, F. Colonna, and A. Savin, Phys. Rev. A 70, 062505 (2004).

${ }^{12}$ R. Pollet, A. Savin, T. Leininger, and H. Stoll, J. Chem. Phys. 116, 1250 (2002).

${ }^{13}$ T. Leininger, H. Stoll, H.-J. Werner, and A. Savin, Chem. Phys. Lett. 275, 151 (1997).

${ }^{14}$ Y. Tawada, T. Tsuneda, S. Yanagisawa, T. Yanai, and K. Hirao, J. Chem. Phys. 120, 8425 (2004).

${ }^{15}$ H. Iikura, T. Tsuneda, T. Yanai, and K. Hirao, J. Chem. Phys. 115, 3540 (2001).

${ }^{16}$ T. Yanai, D. P. Tew, and N. C. Handy, Chem. Phys. Lett. 393, 51 (2004).

${ }^{17}$ J. P. Perdew, A. Ruzsinszky, L. A. Constantin, J. Sun, and G. I. Csonka, J. Chem.

Theory Comput. 5, 902 (2009).

${ }^{18}$ A. D. Becke, J. Chem. Phys. 98, 5648 (1993).

${ }^{19}$ V. Barone, L. Orlandini, and C. Adamo, Chem. Phys. Lett. 231, 295 (1994). 
${ }^{20}$ P. J. Stephens, F. J. Devlin, C. F. Chabalowski, and M. J. Frisch, J. Phys. Chem. 98, 11623 (1994).

${ }^{21}$ C. Adamo and V. Barone, J. Chem. Phys. 110, 6158 (1999).

${ }^{22}$ M. Ernzerhof and G. E. Scuseria, J. Chem. Phys. 110, 5029 (1999).

${ }^{23}$ A. Dreuw and M. Head-Gordon, J. Am. Chem. Soc. 126, 4007 (2004).

${ }^{24} \mathrm{M}$. Savarese, É. Brémond, and C. Adamo, Theor. Chem. Acc. 135, 99 (2016).

${ }^{25}$ S. N. Steinmann and C. Corminboeuf, J. Chem. Theory Comput. 8, 4305 (2012).

${ }^{26}$ J. Toulouse, W. Zhu, J. G. Ángyán, and A. Savin, Phys. Rev. A 82, 032502 (2010).

${ }^{27}$ É. Brémond, Á. J. Pérez-Jiménez, C. Adamo, and J. C. Sancho-García, J. Chem. Phys. 151, 211104 (2019).

${ }^{28}$ M. Wykes, N. Q. Su, X. Xu, C. Adamo, and J.-C. Sancho-García, J. Chem. Theory Comput. 11, 832 (2015).

${ }^{29}$ M. J. G. Peach, E. I. Tellgren, P. Sałek, T. Helgaker, and D. J. Tozer, J. Phys. Chem. A 111, 11930 (2007).

${ }^{30}$ É. Brémond, M. Savarese, Á. J. Pérez-Jiménez, J. C. Sancho-García, and C. Adamo, J. Phys. Chem. Lett. 6, 3540 (2015).

${ }^{31}$ É. Brémond, M. Savarese, N. Q. Su, Á. J. Pérez-Jiménez, X. Xu, J. C. SanchoGarcía, and C. Adamo, J. Chem. Theory Comput. 12, 459 (2016).

${ }^{32}$ H. S. Yu, S. L. Li, and D. G. Truhlar, J. Chem. Phys. 145, 130901 (2016).

${ }^{33}$ A. D. Becke, J. Chem. Phys. 140, 18A301 (2014).

${ }^{34}$ O. A. Vydrov, G. E. Scuseria, and J. P. Perdew, J. Chem. Phys. 126, 154109 (2007).

${ }^{35}$ O. A. Vydrov, J. Heyd, A. V. Krukau, and G. E. Scuseria, J. Chem. Phys. 125, 074106 (2006).

${ }^{36}$ O. A. Vydrov and G. E. Scuseria, J. Chem. Phys. 125, 234109 (2006).

${ }^{37}$ N. Mardirossian and M. Head-Gordon, Phys. Chem. Chem. Phys. 16, 9904 (2014).

${ }^{38}$ Y.-S. Lin, G.-D. Li, S.-P. Mao, and J.-D. Chai, J. Chem. Theory Comput. 9, 263 (2013).

${ }^{39}$ J.-D. Chai and M. Head-Gordon, Phys. Chem. Chem. Phys. 10, 6615 (2008).

${ }^{40}$ J.-D. Chai and M. Head-Gordon, J. Chem. Phys. 128, 084106 (2008).

${ }^{41}$ P. Verma, B. G. Janesko, Y. Wang, X. He, G. Scalmani, M. J. Frisch, and D. G. Truhlar, J. Chem. Theory Comput. 15, 4804 (2019).

${ }^{42}$ P. Verma, Y. Wang, S. Ghosh, X. He, and D. G. Truhlar, J. Phys. Chem. A 123, 2966 (2019)

${ }^{43}$ R. Peverati and D. G. Truhlar, Phys. Chem. Chem. Phys. 14, 16187 (2012).

${ }^{44}$ R. Peverati and D. G. Truhlar, J. Phys. Chem. Lett. 2, 2810 (2011).

${ }^{45}$ R. Baer, E. Livshits, and U. Salzner, Annu. Rev. Phys. Chem, 61, 85 (2010).

${ }^{46}$ E. Livshits and R. Baer, Phys. Chem. Chem. Phys. 9, 2932 (2007).

${ }^{47}$ T. Koopmans, Physica 1, 104 (1934).

${ }^{48}$ T. Stein, L. Kronik, and R. Baer, J. Am. Chem. Soc. 131, 2818 (2009).

${ }^{49}$ Z. Lin and T. Van Voorhis, J. Chem. Theory Comput. 15, 1226 (2019).

${ }^{50} \mathrm{~J}$. Autschbach and M. Srebro, Acc. Chem. Res. 47, 2592 (2014).

${ }^{51}$ D. Jacquemin, B. Moore, A. Planchat, C. Adamo, and J. Autschbach, J. Chem. Theory Comput. 10, 1677 (2014)

${ }^{52} \mathrm{M}$. Srebro and J. Autschbach, J. Phys. Chem. Lett. 3, 576 (2012).

${ }^{53}$ L. Kronik, T. Stein, S. Refaely-Abramson, and R. Baer, J. Chem. Theory Comput. 8, 1515 (2012).

${ }^{54}$ T. Stein, H. Eisenberg, L. Kronik, and R. Baer, Phys. Rev. Lett. 105, 266802 (2010).

${ }^{55}$ I. Tamblyn, S. Refaely-Abramson, J. B. Neaton, and L. Kronik, J. Phys. Chem. Lett. 5, 2734 (2014).

${ }^{56}$ A. Karolewski, L. Kronik, and S. Kümmel, J. Chem. Phys. 138, 204115 (2013).

${ }^{57}$ É. Brémond, Á. J. Pérez-Jiménez, J. C. Sancho-García, and C. Adamo, J. Chem. Phys. 150, 201102 (2019).
${ }^{58}$ É. Brémond, M. Savarese, Á. J. Pérez-Jiménez, J. C. Sancho-García, and C. Adamo, J. Chem. Theory Comput. 14, 4052 (2018).

${ }^{59}$ J. P. Perdew, K. Burke, and M. Ernzerhof, Phys. Rev. Lett. 77, 3865 (1996).

${ }^{60}$ C. A. Guido, E. Brémond, C. Adamo, and P. Cortona, J. Chem. Phys. 138, 021104 (2013).

${ }^{61}$ E. Brémond and C. Adamo, J. Chem. Phys. 135, 024106 (2011).

${ }^{62}$ É. Brémond, J. C. Sancho-García, Á. J. Pérez-Jiménez, and C. Adamo, J. Chem. Phys. 141, 031101 (2014).

${ }^{63}$ E. Brémond, I. Ciofini, J. C. Sancho-García, and C. Adamo, Acc. Chem. Res. 49, 1503 (2016).

${ }^{64}$ L. Goerigk and S. Grimme, Wiley Interdiscip. Rev.: Comput. Mol. Sci. 4, 576 (2014).

${ }^{65}$ J. C. Sancho-García and C. Adamo, Phys. Chem. Chem. Phys. 15, 14581 (2013).

${ }^{66}$ S. Grimme, J. Chem. Phys. 124, 034108 (2006).

${ }^{67}$ E. Brémond, M. Savarese, C. Adamo, and D. Jacquemin, J. Chem. Theory Comput. 14, 3715 (2018).

${ }^{68}$ L. Goerigk, A. Hansen, C. Bauer, S. Ehrlich, A. Najibi, and S. Grimme, Phys, Chem. Chem. Phys. 19, 32184 (2017).

${ }^{69}$ É. Brémond, M. Savarese, Á. J. Pérez-Jiménez, J. C. Sancho-García, and C. Adamo, J. Chem. Theory Comput. 13, 5539 (2017).

${ }^{70}$ T. Schwabe and L. Goerigk, J. Chem. Theory Comput. 13, 4307 (2017).

${ }^{71}$ D. Bousquet, E. Brémond, J. C. Sancho-García, I. Ciofini, and C. Adamo, J. Chem. Theory Comput. 9, 3444 (2013).

${ }^{72}$ D. W. Smith and O. W. Day, J. Chem. Phys. 62, 113 (1975).

${ }^{73}$ M. M. Morrell, R. G. Parr, and M. Levy, J. Chem. Phys. 62, 549 (1975).

${ }^{74} \mathrm{Y}$. Gu and X. Xu, J. Comput. Chem. 41, 1165 (2020).

${ }^{75}$ J. Cioslowski, P. Piskorz, and G. Liu, J. Chem. Phys. 107, 6804 (1997).

${ }^{76}$ N. Q. Su and X. Xu, J. Phys. Chem. Lett. 10, 2692 (2019).

${ }^{77}$ M. J. Frisch, G. W. Trucks, H. B. Schlegel, G. E. Scuseria, M. A. Robb, J. R. Cheeseman, G. Scalmani, V. Barone, G. A. Petersson, H. Nakatsuji, X. Li, M. Caricato, A. V. Marenich, J. Bloino, B. G. Janesko, R. Gomperts, B. Mennucci, H. P. Hratchian, J. V. Ortiz, A. F. Izmaylov, J. L. Sonnenberg, D. WilliamsYoung, F. Ding, F. Lipparini, F. Egidi, J. Goings, B. Peng, A. Petrone, T. Henderson, D. Ranasinghe, V. G. Zakrzewski, J. Gao, N. Rega, G. Zheng, W. Liang, M. Hada, M. Ehara, K. Toyota, R. Fukuda, J. Hasegawa, M. Ishida, T. Nakajima, Y. Honda, O. Kitao, H. Nakai, T. Vreven, K. Throssell, J. A. Montgomery, Jr., J. E. Peralta, F. Ogliaro, M. J. Bearpark, J. J. Heyd, E. N. Brothers, K. N. Kudin, V. N. Staroverov, T. A. Keith, R. Kobayashi, J. Normand, K. Raghavachari, A. P. Rendell, J. C. Burant, S. S. Iyengar, J. Tomasi, M. Cossi, J. M. Millam, M. Klene, C. Adamo, R. Cammi, J. W. Ochterski, R. L. Martin, K. Morokuma, O. Farkas, J. B. Foresman, and D. J. Fox, Gaussian 16 Revision B.01, Gaussian Inc., Wallingford, CT, 2016.

${ }^{78}$ F. Weigend and R. Ahlrichs, Phys. Chem. Chem. Phys. 7, 3297 (2005).

${ }^{79}$ M. J. van Setten, F. Caruso, S. Sharifzadeh, X. Ren, M. Scheffler, F. Liu, J. Lischner, L. Lin, J. R. Deslippe, S. G. Louie, C. Yang, F. Weigend, J. B. Neaton, F. Evers, and P. Rinke, J. Chem. Theory Comput. 11, 5665 (2015).

${ }^{80}$ K. Krause, M. E. Harding, and W. Klopper, Mol. Phys. 113, 1952 (2015).

${ }^{81}$ J. L. Bao, Y. Wang, X. He, L. Gagliardi, and D. G. Truhlar, J. Phys. Chem. Lett. 8, 5616 (2017).

${ }^{82}$ T. H. Dunning, K. A. Peterson, and D. E. Woon, Encyclopedia of Computational Chemistry (John Wiley \& Sons, Ltd, 2002).

${ }^{83}$ A. Ruzsinszky, J. P. Perdew, G. I. Csonka, O. A. Vydrov, and G. E. Scuseria, J. Chem. Phys. 125, 194112 (2006).

${ }^{84}$ A. D. Dutoi and M. Head-Gordon, Chem. Phys. Lett. 422, 230 (2006).

${ }^{85}$ J. P. Perdew, R. G. Parr, M. Levy, and J. L. Balduz, Phys. Rev. Lett. 49, 1691 (1982). 